\title{
Metachronous, Ipsilateral Primary Lung Cancer: A Case Report
}

\author{
Joseph L. Blidgen, Patrice A. Pinkney \\ Department of Surgery, National Chest Hospital, Kingston, Jamaica \\ Email: josey702001@yahoo.com
}

How to cite this paper: Blidgen, J.L. and Pinkney, P.A. (2021) Metachronous, Ipsilateral Primary Lung Cancer: A Case Report. Journal of Cancer Therapy, 12, 65-70. https://doi.org/10.4236/jct.2021.121007

Received: December 21, 2020

Accepted: January 22, 2021

Published: January 25, 2021

Copyright $\odot 2021$ by author(s) and Scientific Research Publishing Inc. This work is licensed under the Creative Commons Attribution International License (CC BY 4.0).

http://creativecommons.org/licenses/by/4.0/

\begin{abstract}
Lung cancer is the most common malignant tumor in men worldwide. It has been documented that patients can develop multiple primary lung cancer, with a $5 \%$ annual risk of a second primary (metachronous) cancer occurring after prior curative treatment. Moreover, there has been an increase in the incidence of metachronous primary lung cancer reflecting a longer survival after curative resection of the primary cancer and improvements in patient surveillance methods. Herein, we present a case of a patient diagnosed with a metachronous primary lung cancer presenting 1 year after treatment of a primary lung cancer at National Chest Hospital, Kingston, Jamaica. Through strict adherence to surveillance guidelines, this metachronous cancer was detected, enabling the patient to be promptly treated.
\end{abstract}

\section{Keywords}

Lung Cancer, Adenocarcinoma, Metachronous, Jamaica

\section{Introduction}

Lung cancer is the most common malignant tumor worldwide and is the leading cause of cancer mortality [1]. In Jamaica, it accounts for $7.2 \%$ of all new cancers [2]. The development of a primary lung cancer following curative resection of a previous primary lung cancer is referred to as metachronous second primary lung cancer (MSPLC). Every patient who has had curative resection for primary lung cancer may potentially develop MSPLC. Nevertheless, MSPLC is still relatively uncommon compared with second primary tumours in other paired organs such as breast and ovary. With the widespread use of imaging in the form of high resolution computed tomography and positron emission tomography (PET), improvements in surgical cancer care, and longer survival of patients post resection of primary lung cancer, the detection of MSPLC is increasing [3]. Most 
lung cancer deaths occur within 3 years of treatment and thus in the past a subclinical MSPLC would not have been detected. However, high resolution imaging has allowed for the early detection of these MSPLC which are potentially curable if diagnosed at an early stage. Surgical resection remains the mainstay of treatment of MSPLC, with lobectomy considered the goal standard.

Patients who present with MSPLC are usually older with medical comorbidities. They may have poor cardiopulmonary reserve and hence are unable to tolerate a lobectomy but may benefit from a sublobar resection. This has resulted in some controversy regarding optimal surgery for these patients.

We herein present a case report of a patient with adenocarcinoma of the lung that through adherence to follow-up surveillance guidelines was later diagnosed with an MSLPC.

\section{Case Report}

A 57-year-old male with a 20-pack year smoking history presented to the $\mathrm{Na}$ tional Chest Hospital, Kingston, Jamaica with a 7-month history of hemoptysis. His physical examination was normal. Computed Tomography (CT) of the chest revealed a $6.3 \times 5.4 \times 4.4 \mathrm{~cm}$ mass in the apex of the right upper lobe, involving the pleura with no mediastinal lymphadenopathy (Figure 1). Fiber-optic bronchoscopy revealed blood within the right upper lobe bronchus but no endobronchial lesions. Transthoracic percutaneous biopsy revealed an adenocarcinoma histology. Pulmonary function tests were as follows: FVC 3.89L (98\% of predicted) and FEV1 2.61L (82\% of predicted).

Cervical mediastinoscopy yielded a station $4 \mathrm{R}$ lymph node, which was negative for malignancy. He underwent a right upper lobectomy and mediastinal lymph node sampling. Histopathology confirmed poorly differentiated adenocarcinoma with the pleural within $1 \mathrm{~mm}$ of the tumor. Due to the size of the lesion and proximity of the cancer to the pleural surface, he was referred to the oncologist for adjuvant chemotherapy. He received a total of 4 doses of carboplatin and Taxol every 3 weeks.

During surveillance, 13 months later, chest CT scan revealed a $3.9 \times 4.2 \mathrm{~cm}$ mass in the right lower lobe (Figure 2). No mediastinal lymphadenopathy was noted. PET scan revealed a standard uptake for the lung mass and no evidence of mediastinal or extra-thoracic metastases. Repeat pulmonary function tests were as follows: FVC 3.05L (81\% of predicted) and FEV1 3.01L (99\% of predicted). No endobronchial lesion was seen on bronchoscopy. The patient was presumed to have metastatic disease and underwent a right thoracotomy and right lower lobe wedge resection. Histopathology confirmed a poorly differentiated squamous cell carcinoma. The patient was referred to oncology service post operatively and he was advised to have chemo-radiation. He however defaulted. He was admitted to another institution 2 years later with a cerebrovascular accident, which was presumed to be due to metastatic disease and subsequently died. Of note, he died before a CT scan or MRI of the brain was done. Although a postmortem 


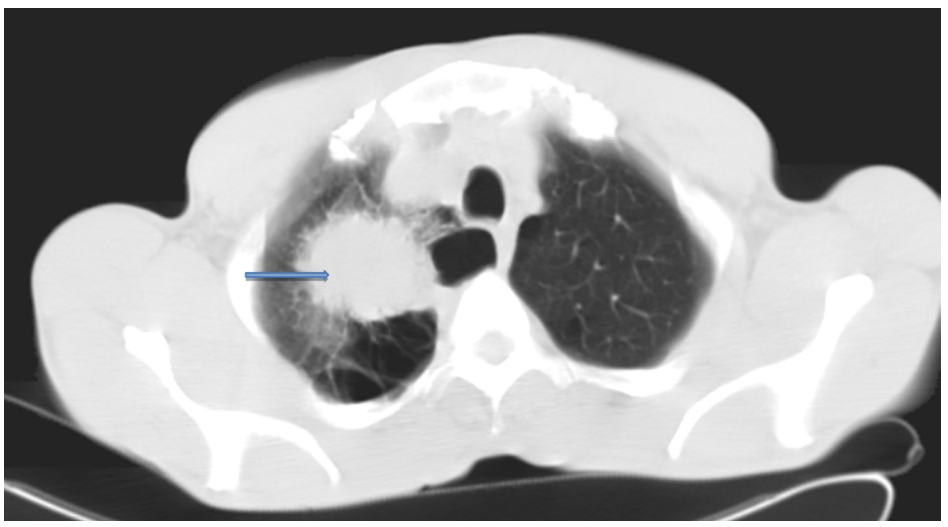

Figure 1. CT showing right upper lobe mass with bullous disease.

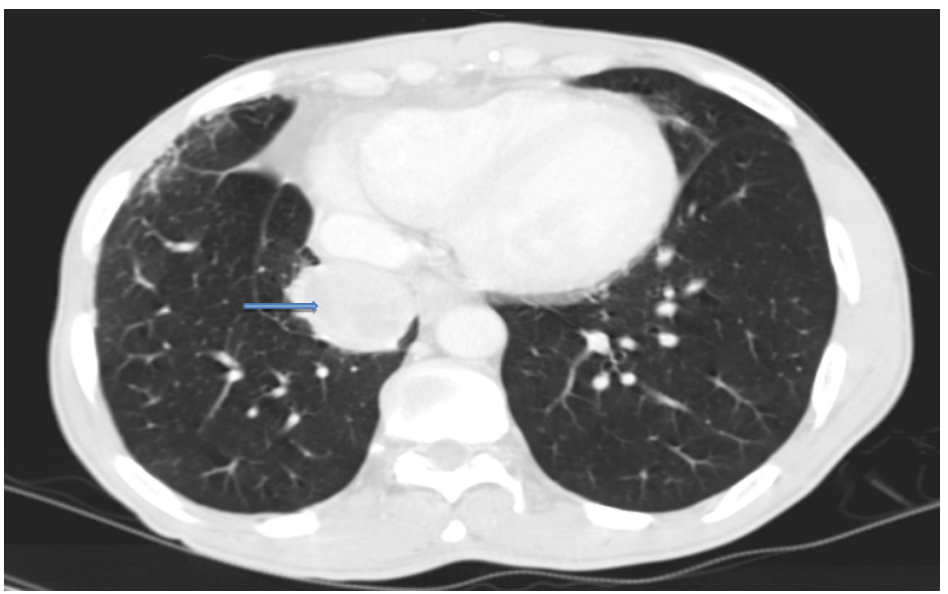

Figure 2. Surveillance CT showing right lower lobe mass 13 months later.

was not performed, based on his presentation and history of non-small cell lung cancer, it is likely that this was a brain metastasis. Though desired, the patient did not have a pre-operative percutaneous transthoracic biopsy performed prior to the second thoracotomy and resection due to the short disease-free interval and suspicion of likely metastatic disease and due to logistical issues. While currently multidisciplinary team (MDT) tumor board meetings are an important part in the management of patients at our hospital, at the time of surgical intervention this was not established at our institution.

\section{Discussion}

Multiple primary lung cancer was first reported in 1924 and has a prevalence of $0.8 \%-14.5 \%$ [4]. MSPLC accounts for 55\% - 65\% of all presentation of multiple primary lung cancer [4]. The lifelong rate of developing a MSPLC is $1 \%-2 \%$ and $6 \%$ per year after radical treatment of non-small cell lung cancer (NSCLC) and small cell lung cancer (SCLC) respectively [5].

It is difficult to distinguish MSPLC from a recurrence or metastatic disease arising from the first tumor. This distinction is important because prognosis and treatment are drastically different. In patients who develop recurrent disease, sur- 
vival is $51.8 \%$ at 2 years in patients with a MSPLC compared to $24.3 \%$ in those with local recurrence and 8.9\% in those with nonregional metastases [6]. In 1975 Martini and Melamed proposed criteria to assist with the identification of a likely MSPLC [7]. They proposed that if the histology is different, as in our index case, it is likely to be a MSPLC. If the histology is the same but the cancer-free interval between cancers is at least 2 years or if the origin is from carcinoma-insitu or if it is a second cancer in a different lobe or lung but no carcinoma in lymphatics common to both or no extrapulmonary metastasis at time of diagnosis, then it is likely to be a MSPLC [7]. These criteria have provided the best treatment guidance for surgical management of patients with MSPLC. In addition, a number of molecular techniques such as p53 mutation analysis have been used to distinguish MSPLC from recurrences of the original tumour [8]. The importance of distinguishing MSPLC from a recurrence or metastases is the ability to offer treatment with curative intent.

Long-term follow-up is necessary for all patients who have undergone resection of primary NSCLC and is the mainstay of diagnosis of MPSLC. In some series $68 \%$ to $100 \%$ of patients with MPSLC were asymptomatic and had the new primary cancer detected by radiographic methods. While there are guidelines providing specific recommendations for surveillance methods in patients treated for NSCLC, there is currently no consensus regarding chest imaging. The European Society for Medical Oncology guidelines does not recommend any chest imaging; however, the American College of Radiology recommends a chest radiograph every 2 to 4 months and CT scan every 12 months whereas the American College of chest physician recommends chest radiograph or CT scan every 6 months. In Jamaica, post curative resection chest CT scans are done every 6 months.

The mean 5-year survival in patients who can undergo resection is $36 \%(20 \%-$ 50\%) [7] [9]. Surgical treatment depends on age, the site and size of the newly developed tumor, the surgical procedure performed on the first tumor and the patient's pulmonary function [8]. Once extrapulmonary metastases are excluded and the patient can tolerate surgery, then surgical resection remains the most effective treatment for patients with MSPLC.

Some surgeons reported that sublobar resection is acceptable for MSPLC whereas others consider that anatomic resection is favorable [10] [11]. In our patient, a sublobar resection was done as the lesion was initially thought to be metastatic. Additionally, a sublobar resection is an acceptable treatment for MSPLC with satisfactory survival. For stage 1 disease, lobectomy tends to be associated with a better survival than sublobar resection, although the difference is not significant [12]. In contrast, other surgical series have reported that the type of resection for MSPLC did not predict survival [13] [14]. With limited or restricted pulmonary function, a sub-lobar resection should be performed. In cases where resection is not possible chemotherapy, radiation or a combination is advocated. Additionally, in our patient, a sublobar resection allowed for preservation of lung 
function.

This case revealed the challenges associated with the management of any patient with lung cancer and incorporation of guidelines in establishing a diagnosis. An integral component of Martini and Melamed's guideline is a disease-free interval of greater two years for the diagnosis of MSPLC. The index patient however was diagnosed at 13 months. Given the relatively short timeline, pulmonary metastasis of the initial lung cancer was our primary diagnosis. The possibility of a new primary was considered but was thought to be unlikely and lack of a preoperative biopsy affected our decision making. The incorporation of the Martini and Melamed's guidelines allows for the accurate differentiation of MSPLC from recurrences or metastases which enables patients with MSPLC to have curative treatment. A further clinical challenge was the lack of compliance of our patient to recommended adjuvant chemo-radiation after the second resection. This may have contributed to his poor survival.

MSPLC should be a differential in all patients noted to have new lesions on surveillance scans. Patients should be considered for resection once they have no evidence of systemic metastasis and adequate pulmonary function.

\section{Conflicts of Interest}

The authors declare no conflicts of interest regarding the publication of this paper.

\section{References}

[1] Bray, F., Ferlay, J., Soerjomataram, I., Siegel, R.L., Torre, L.A. and Jemal, A. (2018) Global Cancer Statistics 2018: GLOBOCAN Estimates of Incidence and Mortality Worldwide for 36 Cancers in 185 Countries. CA: A Cancer Journal for Clinicians, 68, 394-424. https://doi.org/10.3322/caac.21492

[2] Ferlay, J., Ervik, M., Lam, F., Colombet, M., Mery, L., Piñeros, M., Znaor, A., Soerjomataram, I. and Bray, F. Global Cancer Observatory: Cancer Today. International Agency for Research on Cancer, Lyon. https://gco.iarc.fr/today

[3] Rubins, J., Unger, M. and Colice, G.L. (2007) American College of Chest P. Follow-Up and Surveillance of the Lung Cancer Patient Following Curative Intent Therapy: ACCP Evidence-Based Clinical Practice Guideline (2nd Edition). Chest, 132, 355S-367S. https://doi.org/10.1378/chest.07-1390

[4] Adebonojo, S.A., Moritz, D.M. and Danby, C.A. (1997) The Results of Modern Surgical Therapy for Multiple Primary Lung Cancers. Chest, 112, 693-701.

https://doi.org/10.1378/chest.112.3.693

[5] Johnson, B.E. (1998) Second Lung Cancers in Patients after Treatment for an Initial Lung Cancer. Journal of the National Cancer Institute, 90, 1335-1345. https://doi.org/10.1093/jnci/90.18.1335

[6] Pairolero, P.C., Williams, D.E., Bergstralh, E.J., Piehler, J.M., Bernatz, P.E. and Payne, W.S. (1984) Postsurgical Stage I Bronchogenic Carcinoma: Morbid Implications of Recurrent Disease. The Annals of Thoracic Surgery, 38, 331-338. https://doi.org/10.1016/S0003-4975(10)62281-3

[7] Martini, N. and Melamed, M.R. (1975) Multiple Primary Lung Cancers. The Journal of Thoracic and Cardiovascular Surgery, 70, 606-612. 
https://doi.org/10.1016/S0022-5223(19)40289-4

[8] van Rens, M.Th.M., Eijken, E.J.E., Elbers, J.R.J., Lammers, J.-W.J., Tilanus, M.G.J. and Slootweg, P.J. (2002) p53 Mutation Analysis for Definite Diagnosis of Multiple Primary Lung Carcinoma. Cancer, 94, 188-196. https://doi.org/10.1002/cncr.10001

[9] Shen, K.R., Meyers, B.F., Larner, J.M. and Jones, D.R. (2007) American College of Chest P. Special Treatment Issues in Lung Cancer: ACCP Evidence-Based Clinical Practice Guidelines (2nd Edition). Chest, 132, 290S-305S. https://doi.org/10.1378/chest.07-1382

[10] Zuin, A., Andriolo, L.G., Marulli, G., Schiavon, M., Nicotra, S., Calabrese, F., et al. (2013) Is Lobectomy Really More Effective than Sublobar Resection in the Surgical Treatment of Second Primary Lung Cancer? European Journal of Cardio-Thoracic Surgery, 44, e120-125. https://doi.org/10.1093/ejcts/ezt219

[11] Doddoli, C., Thomas, P., Ghez, O., Giudicelli, R. and Fuentes, P. (2001) Surgical Management of Metachronous Bronchial Carcinoma. European Journal of CardioThoracic Surgery, 19, 899-903. https://doi.org/10.1016/S1010-7940(01)00690-X

[12] Yang, J., Liu, M., Fan, J., Song, N., He, W.X., Yang, Y.L., et al. (2014) Surgical Treatment of Metachronous Second Primary Lung Cancer. The Annals of Thoracic Surgery, 98, 1192-1198. https://doi.org/10.1016/j.athoracsur.2014.05.050

[13] Battafarano, R.J., Force, S.D., Meyers, B.F., Bell, J., Guthrie, T.J., Cooper, J.D., et al. (2004) Benefits of Resection for Metachronous Lung Cancer. The Journal of Thoracic and Cardiovascular Surgery, 127, 836-842. https://doi.org/10.1016/j.jtcvs.2003.08.055

[14] Aziz, T.M., Saad, R.A., Glasser, J., Jilaihawi, A.N. and Prakash, D. (2002) The Management of Second Primary Lung Cancers. A Single Centre Experience in 15 Years. European Journal of Cardio-Thoracic Surgery, 21, 527-533. https://doi.org/10.1016/S1010-7940(02)00024-6 